\title{
Theory of elasticity of the Abrikosov flux-line lattice for uniaxial superconductors: Parallel flux lines
}

\author{
Edson Sardella* \\ Universidade Estadual Paulista Julio de Mesquita Filho, Campus de Ilha Solteira, \\ Ilha Solteira-São Paulo, Cep 15378, Brazil
}

(Received 19 November 1990)

\begin{abstract}
In this paper, we consider the extension of the Brandt theory of elasticity of the Abrikosov flux-line lattice for a uniaxial superconductor for the case of parallel flux lines. The results show that the effect of the anisotropy is to rescale the components of the wave vector $\mathbf{k}$ and the magnetic field and orderparameter wave vector cut off by a geometrical parameter previously introduced by Kogan.
\end{abstract}

\section{INTRODUCTION}

It is well known that the high- $T_{c}$ superconductors are strongly anisotropic compounds. These materials can be described by the Ginzburg-Landau (GL) equations in the anisotropic form which involve the principal values $M_{i}$ ( $i=X, Y, Z)$ of the effective mass tensor $M_{i j}$. The high$T_{c}$ superconductors are uniaxial (or nearly so) compounds, so that we can take $M_{X}=M_{Y} \neq M_{Z}$, where $M_{Z}$ is the effective mass along the $Z$ direction (perpendicular to the layer). The energy cost displacing the flux lines of a uniaxial superconductor from their equilibrium configuration is the major aim of this paper.

This effective-mass model can well describe the angular dependence of the upper critical field. However, some recent investigations ${ }^{1}$ have pointed out that the effectivemass model cannot account for some unusual physical properties of the strong anisotropic superconductors at a microscopic level. Therefore, the expression "high- $T_{c}$ superconductor" should be carefully interpreted throughout this paper.

This paper is organized as follows. In Sec. II we present the Abrikosov solution of the anisotropic linear GL equations in a different (but equivalent) form from those addressed in some previous works. ${ }^{2-4}$ In this section no essential new result is presented. However, the way in which we treat the problem will be much more convenient for our purpose, because it is valid even for a distorted flux-line lattice. In Sec. III we then show how the Abrikosov solution fails for a uniaxial superconductor in the short wave-vector limit. Finally, in Sec. IV we remove the divergence of the order parameter and magnetic field by using the same procedure as Brandt did in his pioneering work ${ }^{5}$ for isotropic superconductors.

\section{THE ABRIKOSOV SOLUTION}

The starting point of the calculation is the GL phenomenological free energy which in reduced units can be written as ${ }^{6}$

$$
\begin{gathered}
F=\int d V\left[\sum_{i, j} \mu_{i j}\left[\frac{1}{i \kappa} \frac{\partial}{\partial X_{i}}-A_{i}\right] \Psi\left[\frac{i}{\kappa} \frac{\partial}{\partial X_{i}}-A_{i}\right] \Psi^{*}\right. \\
\left.+\frac{1}{2}|\Psi|^{4}-|\Psi|^{2}+H^{2}\right]
\end{gathered}
$$

where $d V=d X d Y d Z$ is the element of volume, $\kappa$ is the GL parameter, and $\mu_{X X}=\mu_{Y Y}=1, \mu_{Z Z}=M / M_{Z}=\epsilon$ and zero otherwise; $M_{X}=M_{Y}=M ; \mathbf{H}=\nabla \times \mathbf{A}$, where $\mathbf{A}$ is the vector potential.

Let us rotate the crystal frame $(X, Y, Z)$ through an angle $\theta$ about the $Y$ axis onto the vortex frame $(x, y, z)$ (see Fig. 1 of Ref. 2). It can be easily shown that Eq. (1) has the same form in the new system of coordinates with $X_{i}$ replaced by $x_{i}$ and $\mu_{i j}$ by $^{2}$

$$
\begin{aligned}
& \mu_{x x}=\cos ^{2} \theta+\epsilon \sin ^{2} \theta=\gamma^{4}, \\
& \mu_{y y}=1, \mu_{x y}=\mu_{y z}=0, \\
& \mu_{z z}=\sin ^{2} \theta+\epsilon \cos ^{2} \theta, \\
& \mu_{x z}=(1-\epsilon) \sin \theta \cos \theta .
\end{aligned}
$$

Near the upper critical field, the usual procedure is to solve the eigenvalue $(\lambda)$-eigenvector $\left(\Psi_{l}\right)$ linear GL equation,

$\sum_{i, j} \mu_{i j}\left(\frac{1}{i \kappa} \frac{\partial}{\partial x_{i}}-A_{i}\right)\left(\frac{1}{i \kappa} \frac{\partial}{\partial x_{j}}-A_{j}\right) \Psi_{l}-\Psi_{l}=\lambda \Psi_{l}$,

with $\mathbf{A}=\mathbf{A}_{B}=(B / 2) \widehat{\mathbf{z}} \times \mathbf{r}, \quad \mathbf{B}=\langle\mathbf{H}\rangle \widehat{\mathbf{z}}$, where $\langle\cdots\rangle$ denotes a spatial average.

Let us assume that all quantities in the vortex frame are $z$ independent. In this case, Eq. (3) becomes

$\left[\gamma^{4}\left[\frac{1}{i \kappa} \frac{\partial}{\partial x}+\frac{B}{2} y\right]^{2}+\left[\frac{1}{i \kappa} \frac{\partial}{\partial y}-\frac{B}{2} x\right]^{2}-1\right] \Psi_{l}=\lambda \Psi_{l}$.

In order to solve this equation, we first rewrite Eq. (4) in terms of the creation and destruction operators $F_{+}$ and $F_{-}$, respectively,

$$
\left[\left(\widetilde{B} / \gamma^{2} \widetilde{\kappa}-1\right)+\frac{1}{\gamma^{2}} F_{+} F_{-}\right] \Psi_{l}=\lambda \Psi_{l},
$$

where

$$
F_{ \pm}=\gamma \frac{1}{i \widetilde{\kappa}} \frac{\partial}{\partial x} \mp \frac{1}{\gamma i \widetilde{\kappa}} \frac{\partial}{\partial y} \mp \frac{\widetilde{B}}{2 i} \frac{x}{\gamma}+\frac{\widetilde{B}}{2 i} \gamma y,
$$

and $\widetilde{\boldsymbol{\kappa}}=\kappa / \gamma^{2}, \widetilde{\boldsymbol{B}}=\gamma^{2} \boldsymbol{B}$.

For magnetic fields close to $H_{c 2}$ it is sufficient to keep 
only the lowest eigenvalue $\lambda=\left(\widetilde{B} / \gamma^{2} \widetilde{\boldsymbol{\kappa}}-1\right)$ which corresponds to $F_{-} \Psi_{l}=0$. The solution of this equation may be found by replacing $(x, y), B$, and $\kappa$ in the isotropic case by $(x / \gamma, \gamma y), \widetilde{B}$, and $\widetilde{\kappa}$. We then obtain

$\Psi_{l}^{(0)}(x, y)=C \exp \left[-\left(x^{2} / \gamma^{2}+\gamma^{2} y^{2}\right) \frac{\widetilde{B} \widetilde{\kappa}}{4}\right] g(x / \gamma+i \gamma y)$,

where $g(x / \gamma+i \gamma y)$ is some function which produces the correct zeros of $\omega_{l}=\left|\Psi_{l}^{(0)}\right|^{2}$ and $C$ is a constant of normalization. It has been demonstrated by $\operatorname{Kogan}^{7}$ that this function can be taken as the product of all $\left(x-x_{v}\right) / \gamma+i \gamma\left(y-y_{v}\right)$ where $\left(x_{v}, y_{v}\right)$ is the position of the $v$ th flux line. Hence, we can write

$$
\begin{aligned}
\omega_{l}(x, y)= & C \exp \left[-\left(x^{2} / \gamma^{2}+\gamma^{2} y^{2}\right) \frac{\widetilde{B} \widetilde{\kappa}}{4}\right] \\
& \times \prod_{v}\left[\left(x-x_{v}\right)^{2} / \gamma^{2}+\gamma^{2}\left(y-y_{v}\right)^{2}\right], \\
\phi_{l}(x, y)= & \sum_{v} \arctan \left[\frac{\left(y-y_{v}\right)^{2} \gamma^{2}}{\left(x-x_{v}\right)^{2}}\right]+\text { const },
\end{aligned}
$$

where $\phi_{l}$ is the phase of $\Psi_{l}^{(0)}$.

Let us now define the supervelocity as $\mathbf{Q}_{B}$ $=\mathbf{A}_{B}-(1 / \kappa) \nabla \phi_{l}$. Inserting Eq. (9) into this definition we find

$\mathbf{Q}_{B}(x, y)=\frac{B}{2} \hat{\mathbf{z}} \times \mathbf{r}-\frac{1}{\kappa} \sum_{v} \frac{\hat{\mathbf{z}} \times\left(\mathbf{r}-\mathbf{r}_{v}\right)}{\left[\left(x-x_{v}\right)^{2} / \gamma^{2}+\gamma^{2}\left(y-y_{v}\right)^{2}\right]}$.

By taking the gradient of Eq. (8) we will have the expressions

$\frac{1}{\omega_{l}} \frac{\partial \omega_{l}}{\partial x}=-\widetilde{B} \widetilde{\kappa} \frac{x}{\gamma^{2}}+2 \sum_{v} \frac{\left(x-x_{v}\right) / \gamma^{2}}{\left[\left(x-x_{v}\right)^{2} / \gamma^{2}+\gamma^{2}\left(y-y_{v}\right)^{2}\right]}$,

$\frac{1}{\omega_{l}} \frac{\partial \omega_{l}}{\partial y}=-\widetilde{\boldsymbol{B}} \widetilde{\kappa} \gamma^{2} y+2 \sum_{v} \frac{\gamma^{2}\left(y-y_{v}\right)^{2}}{\left[\left(x-x_{v}\right)^{2} / \gamma^{2}+\gamma^{2}\left(y-y_{v}\right)^{2}\right]}$.

The combination of Eqs. (10) and (11) yields

$$
Q_{B}=-\widehat{\mathbf{z}} \times\left[\frac{\partial \omega_{l}}{\partial x} \widehat{\mathbf{x}}+\frac{1}{\gamma^{4}} \frac{\partial \omega_{l}}{\partial y} \hat{\mathbf{y}}\right] / 2 \widetilde{\kappa} \omega_{l} .
$$

A straightforward manipulation of the second GL equation and Eq. (12) yields the well-known results

$$
\begin{aligned}
& H_{z}=B+\frac{\left\langle\omega_{l}\right\rangle-\omega_{l}}{2 \widetilde{\kappa}}, \\
& \frac{\partial H_{y}}{\partial x}-\frac{\partial H_{x}}{\partial y}=-\widetilde{\gamma} \frac{\partial \omega_{l}}{\partial y},
\end{aligned}
$$

where $\widetilde{\gamma}=\mu_{x z} / 2 \widetilde{\kappa} \gamma^{4}$. This equation for the transverse field was previously found by Kogan and $\mathrm{Clem}^{2}$ by using a different approach. They have interpreted $\mathbf{H}_{\perp}$ as a consequence of a current which flows along the vortex, even when the system approaches $H_{c 2}$.

Let us mention some features of the present derivation.

(1) It can be extended to the case in which the flux lines are displaced from their equilibrium positions. This situation is of fundamental importance to the theory of elasticity of the flux-line lattice.

(2) The equilibrium lattice structure can be determined. As can be seen in Eq. (7), the order parameter appears with $x$ and $y$ rescaled by $1 / \gamma$ and $\gamma$, respectively. This means that if the structure of the lattice for the isotropic superconductor is a triangular lattice with periods $(1,0)$ and $(1 / 2, \sqrt{3} / 2)$, then that for an anisotropic superconductor will be $(\gamma, 0)$ and $(\gamma / 2, \sqrt{3} / 2 \gamma)$. Campbell et al., ${ }^{8}$ in a very recent study of the structure of a uniaxial layered crystal, have found the same result by working out the GL free energy in the London regime. This result has also been confirmed by Petzinger and Warren ${ }^{4}$ by using a better sophisticated mathematical apparatus than the present one.

(3) As can be seen from Eq. (8), $\omega_{l}$ (and consequently the free energy) is not invariant under rotation about the $z$ axis. This new feature of the free energy also occurs in the London regime. ${ }^{9}$

\section{DIVERGENCE OF $\omega_{l}$ AND H}

Up to this point we have not taken into account any distortion of the flux-line lattice. However, as was observed in Sec. II, our previous derivation of Eqs. (7)-(14) is still valid even when the flux lines are displaced from their equilibrium positions. Let us denote by $\mathbf{s}_{v}=\left(s_{v}^{x}, s_{v}^{y}, z\right)$ the displacement of the $v$ th flux line from the initial regular flux position $\mathbf{R}_{v}=\left(X_{v}, Y_{v}, z\right)$ parallel to the $z$ axes. If we introduce $\mathbf{r}_{v}=\mathbf{R}_{v}+\mathbf{s}_{v}$ in Eq. (8) and then expand it in powers of $\mathbf{s}_{v}$, we obtain

$$
\omega_{l}(x, y)=\omega_{A}(x, y)(1+\eta / 2)^{2}+o\left(s^{2}\right),
$$

where

$\eta(x, y)=-2 \sum_{v} \frac{s_{v}^{x}\left(x-x_{v}\right) / \gamma^{2}+\gamma^{2} s_{v}^{y}\left(y-y_{v}\right)}{\left[\left(x-x_{v}\right)^{2} / \gamma^{2}+\gamma^{2}\left(y-y_{v}\right)^{2}\right]}$,

and $\omega_{A}(x, y)$ is given by Eq. (8) with $\mathbf{r}_{v}=\mathbf{R}_{v}$.

By taking a periodic displacement field

$$
\mathbf{s}_{v}=\operatorname{Re}\left(\mathbf{s}_{0} e^{i \mathbf{k} \cdot \mathbf{R}_{v}}\right) \text {, }
$$

with $\mathbf{k}=\left(k_{x}, k_{y}, 0\right)$, in Appendix A we find

$$
\eta(x, y)=\operatorname{Re}\left[2 \widetilde{B} \widetilde{\kappa} \sum_{\mathbf{K}} \frac{i(\mathbf{k}+\mathbf{K}) \cdot \mathbf{s}_{0} e^{i(\mathbf{k}+\mathbf{K}) \cdot \mathbf{r}}}{\left[\gamma^{2}\left(k_{x}+K_{x}\right)^{2}+\left(k_{y}+K_{y}\right)^{2} / \gamma^{2}\right]}\right],
$$

where $\mathbf{K}$ is the reciprocal lattice vector and is given by ${ }^{8}$ $\mathbf{K}=m \mathbf{b}_{1}+n \mathbf{b}_{2} \quad$ with $\quad \mathbf{b}_{1}=(2 \pi / \sqrt{3})[(\sqrt{3} / \gamma) \hat{\mathbf{x}}-\gamma \hat{\mathbf{y}}]$, $\mathbf{b}_{2}=(4 \pi / \sqrt{3}) \gamma \hat{\mathbf{y}}$ ( $m, n$ integers).

For $k \ll K_{10}$, Eq. (17) may be written in an approximated form as follows: 


$$
\begin{aligned}
\eta(x, y) & \approx \operatorname{Re}\left[2 \widetilde{B} \widetilde{\kappa} \mathbf{s}_{0} \cdot\left[\frac{i \mathbf{k}}{\widetilde{k}^{2}}+\sum_{\mathbf{K} \neq 0} \frac{i \mathbf{K}}{\widetilde{K}^{2}} e^{i \mathbf{K} \cdot \mathbf{r}}\right]\right] \\
& =2 \widetilde{B} \widetilde{\boldsymbol{\kappa}} \frac{\boldsymbol{\nabla} \cdot \mathbf{s}(\mathbf{r})}{\widetilde{k}^{2}}-\frac{\mathbf{s}(\mathbf{r}) \cdot \boldsymbol{\nabla} \omega_{A}(\mathbf{r})}{\omega_{A}(\mathbf{r})},
\end{aligned}
$$

where, on going from the first to the second line, we have used the following identity: ${ }^{5}$

$$
\frac{\nabla \omega_{A}(\mathbf{r})}{\omega_{A}(\mathbf{r})}=-2 \widetilde{B} \widetilde{\kappa} \sum_{\mathbf{K} \neq 0} \frac{i \mathbf{K}}{\widetilde{K}^{2}} e^{i \mathbf{K} \cdot \mathbf{r}},
$$

which can be found from Eq. (11) by taking $\mathbf{r}_{v}=\mathbf{R}_{v}$ and using Eq. (2) of Appendix A. In Eq. (19) $\widetilde{\mathbf{k}}=\left(\gamma k_{x}, k_{y} / \gamma, 0\right)$ and $\mathbf{s}(\mathbf{r})$ is a smooth displacement field given by Eq. (17) with $\mathbf{R}_{v}$ replaced by $\mathbf{r}$.

The substitution of Eq. (19) into Eqs. (13) and (15) gives

$$
\begin{aligned}
\omega_{l}(x, y)= & \omega_{A}[\mathbf{r}-\mathbf{s}(\mathbf{r})]\left[1+2 \widetilde{B} \widetilde{\kappa} \frac{\nabla \cdot \mathbf{s}(\mathbf{r})}{\widetilde{k}^{2}}\right]+o\left(s^{2}\right), \\
H_{z}(x, y)= & B+\frac{\left\langle\omega_{A}\right\rangle-\omega_{A}[\mathbf{r}-\mathbf{s}(\mathbf{r})]}{2 \widetilde{\kappa}} \\
& +\omega_{A}[\mathbf{r}-\mathbf{s}(\mathbf{r})] \widetilde{B} \frac{\nabla \cdot \mathbf{s}(\mathbf{r})}{\widetilde{k}^{2}}+o\left(s^{2}\right)
\end{aligned}
$$

If we compare these results with the isotropic equivalents ${ }^{5}$ we will see that the effect of the anisotropy is to replace $\mathbf{k}, B$, and $\kappa$ by $\widetilde{k}, \widetilde{B}$, and $\widetilde{\kappa}$.

To proceed, let us now solve for $\mathbf{H}_{\perp}$. Operating on both sides of Eq. (14) with $\partial / \partial y$ and using $\boldsymbol{\nabla} \cdot \mathbf{H}=0$ we obtain

$$
\nabla^{2} H_{x}=\widetilde{\gamma} \frac{\partial^{2} \omega_{l}}{\partial y^{2}} .
$$

Similarly we have

$$
\nabla^{2} \boldsymbol{H}_{y}=-\widetilde{\gamma} \frac{\partial^{2} \omega_{l}}{\partial x \partial_{y}}
$$

We will solve Eqs. (23) and (24) by neglecting variations of $\omega_{A}(x, y)$ in Eq. (19), which allows us to take $\eta \approx \bar{\eta}(x, y)=2 \widetilde{B} \widetilde{\kappa} \nabla \cdot \mathbf{s}(\mathbf{r}) / \widetilde{k}^{2}$. This is equivalent to saying that we are interested only in the slowly varying parts of the magnetic field. Brandt ${ }^{5}$ has called this approximation a local average, i.e., only those terms associated with $\mathbf{K}=0$ are considered. Therefore, if one introduces $\omega_{l} \approx \omega_{A}(1+\bar{\eta})$ in Eqs. (23) and (24), by using Fourier transforming one easily obtains

$$
\begin{aligned}
H_{x}(x, y)= & \widetilde{\gamma} \sum_{\mathbf{K} \neq 0} \omega_{\mathbf{K}}\left[\frac{K_{y}^{2}}{K^{2}}+\bar{\eta} \frac{\left(k_{y}+K_{y}^{2}\right)}{(\mathbf{k}+\mathbf{K})^{2}}\right] e^{i \mathbf{K} \cdot \mathbf{r}}+o\left(s^{2}\right) \\
\approx & \widetilde{\gamma} \sum_{\mathbf{K} \neq 0} \frac{K_{y}^{2}}{K^{2}} \omega_{\mathbf{K}}(1+\bar{\eta}), \\
H_{y}(x, y)= & -\widetilde{\gamma} \sum_{\mathbf{K} \neq 0} \omega_{\mathbf{K}}\left[\frac{K_{x} K_{y}}{K^{2}}+\bar{\eta} \frac{\left(k_{x}+K_{x}\right)\left(k_{y}+K_{y}\right)}{(\mathbf{k}+\mathbf{K})^{2}}\right] e^{i \mathbf{K} \cdot \mathbf{r}} \\
& +o\left(s^{2}\right) \approx \widetilde{\gamma} \sum_{\mathbf{K} \neq 0} \frac{K_{x} K_{y}}{K^{2}} \omega_{\mathbf{K}}(1+\bar{\eta}),
\end{aligned}
$$

where $\omega_{\mathbf{K}}$ is the Fourier component of $\omega_{A}(x, y)$.

According to Eqs. (21), (22), (25), and (26), the order parameter and the magnetic field diverge as $1 / \widetilde{k}^{2}$. Brand $\mathrm{t}^{5}$ has removed this unphysical divergence by employing an exhaustive variational method. This question will be left to the next section. ${ }^{10}$

\section{REMOVAL OF THE DIVERGENCE}

In this section we calculate the excess free energy associated with the small displacement field $\mathbf{s}(\mathbf{r})$ of the flux lines. Before we carry this out we still have to perform some preliminary calculations. If one introduces $\Psi=\sqrt{\omega} e^{i \phi}$ in Eq. (1), one obtains

$$
\begin{gathered}
F=\int d v\left[\sum_{i, j} \mu_{i j}\left[\frac{1}{4 \kappa^{2} \omega} \frac{\partial \omega}{\partial x_{i}} \frac{\partial \omega}{\partial x_{j}}+\omega Q_{i} Q_{j}\right]\right. \\
\left.+\frac{1}{2} \omega^{2}-\omega+\mathbf{H}^{2}\right] .
\end{gathered}
$$

Let us define $\mathbf{h}=\mathbf{H}-B \widehat{\mathbf{z}}=\boldsymbol{\nabla} \times\left(\mathbf{A}-\mathbf{A}_{B}\right)=\boldsymbol{\nabla} \times \mathbf{A}_{h}$. Hence, the superfluid velocity is $\mathbf{Q}=\mathbf{A}_{h}+\mathbf{Q}_{B}$ and $\left\langle H^{2}\right\rangle=\left\langle h^{2}\right\rangle+B^{2}$. If we now minimize the free energy with respect to $\mathbf{A}_{h}$ we find

$$
\begin{aligned}
\boldsymbol{\nabla} \times \mathbf{h}=-\omega[ & \left(\gamma^{4} Q_{x}+\mu_{x z} Q_{z}\right) \widehat{\mathbf{x}}+Q_{y} \widehat{\mathbf{y}} \\
& \left.+\left(\mu_{z z} Q_{z}+\mu_{x z} Q_{x}\right) \widehat{\mathbf{z}}\right] .
\end{aligned}
$$

The corresponding minimum free energy can be evaluated by substituting Eq. (28) into Eq. (27),

$$
\begin{gathered}
F=\int d v\left[\sum_{i, j} \mu_{i j}\left[\frac{1}{4 \kappa^{2} \omega} \frac{\partial \omega}{\partial x_{i}} \frac{\partial \omega}{\partial x_{j}}+\omega Q_{B}^{i} Q_{B}^{j}\right]\right. \\
\left.+\frac{1}{2} \omega^{2}-\omega-\mathbf{h}_{B} \cdot \mathbf{h}\right]+B^{2},
\end{gathered}
$$

where $\mathbf{h}_{B}$ obeys the same equation as (28) with $\mathbf{Q}$ replaced by $\mathbf{Q}_{B}$. Notice that Eq. (29) is still valid when the order parameter and the magnetic field are $z$ dependent.

To show how the divergence of the physical properties evaluated in the preceding section can be removed by using a variational technique first proposed by Brandt, ${ }^{5}$ several steps must be taken. First of all, we modulate the amplitude of the order parameter by multiplying the linear solution $\omega_{l}$ by a smooth function $(1+\varphi)$, where $\varphi$ is of first order in $s$. Secondly, we solve the second GL equation for $\mathbf{h}$ and $\mathbf{h}_{B}$. The strategy is to set $\mathbf{h}=\mathbf{h}_{0}+\mathbf{h}_{1}$, $\mathbf{A}_{h}=\mathbf{A}_{0}+\mathbf{A}_{1}$, where $\mathbf{h}_{1}$ and $\mathbf{A}_{1}$ are the fluctuations about the mean field solutions $\mathbf{h}_{0}$ and $\mathbf{A}_{0}$, respectively. At this stage, in addition to those local averages mentioned above, we will make some approximations which consist in neglecting any contribution of the vector potential $\mathbf{A}_{h}$ associated with the transverse field. We will also neglect the fluctuations $\mathbf{h}_{11}$. These approximations will not affect the mean field free energy, just the elastic one. Finally, we evaluate the free energy in terms of $\varphi$ and treat it as a variational parameter.

Having this policy in mind, from Eq. (28) we may write 


$$
Q_{x}=-\frac{1}{\omega} \frac{1}{\gamma^{4}} \frac{\partial h_{z}}{\partial_{y}}, \quad Q_{y}=\frac{1}{\omega} \frac{\partial h_{z}}{\partial x}
$$

If one now substitutes Eq. (30) into ${ }^{11}$

$$
(\boldsymbol{\nabla} \times \mathbf{Q})_{z}=H_{z}-\frac{2 \pi}{\kappa} \sum_{v} \delta_{2}\left(\mathbf{r}-\mathbf{r}_{v}\right)
$$

we obtain

$$
\begin{aligned}
& {\left[\frac{\partial^{2}}{\partial x^{2}}+\frac{1}{\gamma^{4}} \frac{\partial^{2}}{\partial y^{2}}\right] h_{1 z}-\omega h_{1 z}-\frac{\nabla \omega}{\omega} \cdot\left[\hat{\mathbf{x}} \frac{\partial}{\partial x}+\hat{(2)} \frac{1}{\gamma^{4}} \frac{\partial}{\partial y}\right] h_{1 z}} \\
& =\frac{1}{2 \widetilde{\kappa}}\left\{\left[\frac{\partial^{2}}{\partial x^{2}}+\frac{1}{\gamma^{4}} \frac{\partial^{2}}{\partial y^{2}}\right] \omega-\frac{1}{(3)}\left[\left(\frac{\partial \omega}{\partial x}\right]^{2}+\frac{1}{\gamma^{4}}\left[\frac{\partial \omega}{\partial y}\right)^{2}\right]+2 B \widetilde{\kappa} \omega\right\}+\underset{(4)}{\omega h_{0 z}} .
\end{aligned}
$$

Upon using the following identity

$$
\gamma^{2}\left(\frac{\partial \omega_{l}}{\partial x}\right)^{2}+\frac{1}{\gamma^{2}}\left(\frac{\partial \omega_{l}}{\partial y}\right)^{2}-\omega_{l}\left(\gamma^{2} \frac{\partial^{2} \omega_{l}}{\partial x^{2}}+\frac{1}{\gamma^{2}} \frac{\partial^{2} \omega_{l}}{\partial y^{2}}\right)=2 \widetilde{B} \widetilde{\kappa} \omega_{l}^{2},
$$

with $\omega=\omega_{l}(1+\varphi)$, (3) becomes

$$
(3)=\frac{\omega_{l}}{2 \widetilde{\kappa}}\left\{\left[\frac{\partial^{2}}{\partial x^{2}}+\frac{1}{\gamma^{4}} \frac{\partial^{2}}{\partial y^{2}}\right] \varphi-\frac{1}{(1+\varphi)}\left[\left(\frac{\partial \varphi}{\partial x}\right]^{2}+\frac{1}{\gamma^{4}}\left[\frac{\partial \varphi}{\partial y}\right]^{2}\right]\right\} \text {. }
$$

As stated in Sec. III, the approximations which will be used here are $\omega_{A} \approx\left\langle\omega_{A}\right\rangle, \omega \approx\left\langle\omega_{A}\right\rangle(1+\bar{\eta}+\varphi)$. Therefore, up to first order in $s$ we have

$$
\begin{aligned}
& (1) \approx-\langle\omega\rangle h_{1 z}, \\
& (2) \approx 0, \\
& (3) \approx \frac{\langle\omega\rangle}{2 \widetilde{\kappa}}\left[\frac{\partial^{2}}{\partial x^{2}}+\frac{1}{\gamma^{4}} \frac{\partial^{2}}{\partial y^{2}}\right] \varphi, \\
& (4)=\frac{\omega\langle\omega\rangle-\omega^{2}}{2 \widetilde{\kappa}} \approx-\frac{\langle\omega\rangle^{2}}{2 \widetilde{\kappa}}(\bar{\eta}+\varphi),
\end{aligned}
$$

where we have used $\left\langle\omega_{A}\right\rangle=\langle\omega\rangle+o\left(s^{2}\right)$.

Inserted in Eq. (33), these yield

$$
\left[\gamma^{2} \frac{\partial^{2}}{\partial x^{2}}+\frac{1}{\gamma^{2}} \frac{\partial^{2}}{\partial y^{2}}\right] h_{1 z}-\gamma^{2}\langle\omega\rangle h_{1 z}=\frac{\langle\omega\rangle}{2 \widetilde{\kappa}}\left[\left[\gamma^{2} \frac{\partial^{2}}{\partial x^{2}}+\frac{1}{\gamma^{2}} \frac{\partial^{2}}{\partial y^{2}}\right] \varphi-\gamma^{2}\langle\omega\rangle(\bar{\eta}+\varphi)\right] \text {. }
$$

Using the periodicity of $\bar{\eta}, \varphi$, and $h_{1 z}$, we obtain

$$
h_{1 z}=\frac{\langle\omega\rangle}{2 \widetilde{\kappa}}\left[\varphi+\frac{\gamma^{2}\langle\omega\rangle \bar{\eta}}{\widetilde{k}^{2}+\gamma^{2}\langle\omega\rangle}\right) \text {. }
$$

By starting from Eq. (31) with $\mathbf{Q}$ and $H_{z}$ replaced by $\mathbf{Q}_{B}$ and $B$, respectively, a similar method produces

$$
h_{B 1 z}=\frac{\langle\omega\rangle}{2 \widetilde{\kappa}} \varphi \text {. }
$$

Finally, in Appendix B we find for the excess free energy

$$
F_{\mathrm{el}}=F-F_{0}=\frac{1}{4 \widetilde{\kappa}^{2}}\langle\omega\rangle^{2}\left[\left(2 \widetilde{\kappa}^{2}-1\right) \beta+1-2 \widetilde{\kappa}^{2} \widetilde{\gamma}^{2} \beta_{1}(\beta-1)\right]\left\langle(\bar{\eta}+\varphi)^{2}\right\rangle+\frac{\langle\omega\rangle}{4 \widetilde{\kappa}^{2} \gamma^{2}} \widetilde{k}^{2}\left\langle\left[\varphi^{2}-\frac{\tilde{k}_{h}^{2} \bar{\eta}^{2}}{\widetilde{k}^{2}+\widetilde{k}_{h}^{2}}\right]\right\rangle,
$$

where $F_{0}$ is the mean field free energy

$$
F_{0}=-\langle\omega\rangle\left(1-\widetilde{B} / \widetilde{\kappa} \gamma^{2}\right)+\frac{1}{4 \widetilde{\kappa}^{2}}\langle\omega\rangle^{2}\left[\left(2 \widetilde{\kappa}^{2}-1\right) \beta+1-2 \widetilde{\kappa}^{2} \widetilde{\gamma}^{2} \beta_{1}(\beta-1)\right]+B^{2},
$$


$\beta_{1}$ is constant of order unity ${ }^{2}$ [cf. Eq. (B7)], $\beta=\left\langle\omega^{2}\right\rangle /\langle\omega\rangle^{2}$, and $\tilde{k}_{h}^{2}=\gamma^{2}\langle\omega\rangle$.

Now the expressions of $\langle\omega\rangle$ and $\varphi$ which give the minimum of $F_{0}$ and $F_{\mathrm{el}}$ are

$$
\begin{aligned}
& \langle\omega\rangle=\frac{\left(1-\widetilde{B} / \widetilde{\kappa} \gamma^{2}\right) 2 \widetilde{\kappa}^{2}}{\left[\left(2 \widetilde{\kappa}^{2}-1\right) \beta+1-2 \widetilde{\kappa}^{2} \widetilde{\gamma}^{2} \beta_{1}(\beta-1)\right]}, \\
& \varphi=-\frac{\widetilde{k}_{\Psi}^{2} \bar{\eta}}{\widetilde{k}^{2}+\widetilde{k}_{\Psi}^{2}},
\end{aligned}
$$

where

$$
\begin{aligned}
\widetilde{k}_{\Psi}^{2} & =\gamma^{2}\langle\omega\rangle\left[\left(2 \widetilde{\boldsymbol{\kappa}}^{2}-1\right) \beta+1-2 \widetilde{\boldsymbol{\kappa}}^{2} \widetilde{\gamma}^{2} \beta_{1}(\beta-1)\right] \\
& =2 \widetilde{\boldsymbol{\kappa}}^{2} \gamma^{2}\left(1-\widetilde{\boldsymbol{B}} / \widetilde{\boldsymbol{\kappa}} \gamma^{2}\right) \\
& =2 \widetilde{\boldsymbol{\kappa}}^{2} \gamma^{2}(1-B / \widetilde{\boldsymbol{\kappa}}) .
\end{aligned}
$$

Hence, the excess free energy is

$$
F_{\mathrm{el}}=F-F_{0}=\frac{1}{2} c_{L}(\mathbf{k})\left\langle[\boldsymbol{\nabla} \cdot \mathbf{s}(\mathbf{r})]^{2}\right\rangle,
$$

where the compression modulus is

$$
c_{L}(\mathbf{k})=\frac{2 B^{2}\left(1-\tilde{k}_{h}^{2} / \tilde{k}_{\Psi}^{2}\right)}{\left(1+\widetilde{k}^{2} / \tilde{k}_{\Psi}^{2}\right)\left(1+\widetilde{k}^{2} / \tilde{k}_{h}^{2}\right)} .
$$

Notice that the cut off wave vectors $\widetilde{k}_{\Psi}$ and $\widetilde{k}_{h}$ depend on the orientation of the magnetic field. Notice also that they could never be obtained from the equivalent isotropic expressions by a simple substitution of $\kappa$ by an effective $\widetilde{\kappa}$, since they appear rescaled by the geometrical parameter $\gamma$. In addition, in the expression for $\langle\omega\rangle$ appears a new term which depends on the structure of the equilibrium lattice, namely, $2 \widetilde{\kappa}^{2} \widetilde{\gamma}^{2} \beta_{1}(\beta-1)$.

Because $\left\langle\mathbf{H}_{1}\right\rangle=0$, even for anisotropic superconductors, ${ }^{2}$ we have only one compression modulus. This can be easily seen if we write it in the local limit $(\mathbf{k} \rightarrow 0)$ as $^{12}$ $c_{L}=B^{2} \partial^{2} F_{0}(B) / \partial B^{2}$. On the other hand, $c_{L}=c_{11}-c_{66}$, where $c_{66}$ is the shear modulus of the flux lines. There are some indications, both from theoretical and experimental $^{9,13}$ investigation, that suggest there are several $c_{11}$ and $c_{66}$ elastic coefficients. However, their difference is always the same and is given by Eq. (44).

Let us now investigate how the scales of the fluctuations of the order parameter and the magnetic field behave with the orientation of the external magnetic field. Since $\quad \gamma(\theta=0)=1, \quad \gamma(\theta=\pi / 2)=\epsilon^{1 / 4}, \quad \widetilde{\kappa}(\theta=0)=\kappa$, $\widetilde{\kappa}(\theta=\pi / 2)=\kappa / \epsilon^{1 / 2}$, and $\widetilde{\gamma}(\theta=0, \pi / 2)=0$, we have

$$
\begin{aligned}
& \frac{\left(\widetilde{k}_{\Psi}^{2}\right)_{\theta=0}}{\left(\widetilde{k}_{\Psi}^{2}\right)_{\theta=\pi / 2}}=\frac{1-B / H_{c 2}(0)}{1-B / H_{c 2}(\pi / 2)} \epsilon^{1 / 2}, \\
& \frac{\left(\widetilde{k}_{h}^{2}\right)_{\theta=0}}{\left(\widetilde{k}_{h}^{2}\right)_{\theta=\pi / 2}}=\frac{1-B / H_{c 2}(0)}{1-B / H_{c 2}(\pi / 2)} \frac{\left[\left(2 \kappa^{2} / \epsilon-1\right) \beta+1\right]}{\left[\left(2 \kappa^{2}-1\right) \beta+1\right]} \epsilon^{1 / 2}
\end{aligned}
$$

where $H_{c 2}(\theta)=\widetilde{\kappa}(\theta)$. If the intensity of the external magnetic field is such that the ratio $B / H_{c 2}(\theta)$ remains constant for any $\theta$, then

$$
\begin{aligned}
& \frac{\left(\widetilde{k}_{\Psi}^{2}\right)_{\theta=0}}{\left(\widetilde{k}_{\Psi}^{2}\right)_{\theta=\pi / 2}}=\epsilon^{1 / 2}, \\
& \frac{\left(\tilde{k}_{h}^{2}\right)_{\theta=0}}{\left(\tilde{k}_{h}^{2}\right)_{\theta=\pi / 2}}=\frac{\left[\left(2 \kappa^{2} / \epsilon-1\right) \beta+1\right]}{\left[\left(2 \kappa^{2}-1\right) \beta+1\right]} \epsilon^{1 / 2} .
\end{aligned}
$$

For a layered superconductor $\epsilon<1$, so that $\left(\tilde{k}_{\Psi}^{2}\right)_{\theta=0}<\left(\tilde{k}_{\Psi}^{2}\right)_{\theta=\pi / 2}$. For most of the high- $T_{c}$ superconductors $\kappa \gg>1$, which implies $\left[\left(\widetilde{k}_{h}^{2}\right)_{\theta=0} /\left(\tilde{k}_{h}^{2}\right)_{\theta=\pi / 2}\right]$ $\approx 1 / \epsilon^{1 / 2}>1$, or even $\left(\tilde{k}_{h}^{2}\right)_{\theta=0}>\left(\widetilde{k}_{h}^{2}\right)_{\theta=\pi / 2}$. In conclusion, the length scale of the order parameter (magnetic field) is larger (smaller) for the external magnetic field pointing along the $Z$ axis than in the $\mathrm{Cu}-\mathrm{O}$ plane. In other words, as the direction of the applied magnetic field varies continuously from $\theta=0$ to $\theta=\pi / 2$, an increasing of the magnetic field length scale is compensated by a decreasing of the order-parameter length scale.

\section{SUMMARY}

We have extended the Brandt theory of elasticity of the Abrikovos flux-line lattice for a uniaxial superconductor with the external magnetic field in an arbitrary direction. We have also paved the way for the generalization of this theory taking into account the tilting effects (this problem will be left to a future contribution).

\section{ACKNOWLEDGMENTS}

The author is indebted to Professor M. A. Moore for useful discussions. This work was supported by CNPqConselho Nacional de Desemvolvimento Cientifico e Tecnológico-Brazil under process number 200471/88.0.

\section{APPENDIX A}

In order to derive Eq. (18), we need the following identity:

$$
\boldsymbol{\nabla} \cdot\left[\frac{\mathbf{r}}{x^{2} / \gamma^{2}+\gamma^{2} y^{2}}\right]=2 \pi \delta_{2}(\mathbf{r})
$$

which can also be written in an integral form,

$$
\frac{\mathbf{r}}{x^{2} / \gamma^{2}+\gamma^{2} y^{2}}=-\frac{i}{2 \pi} \int d^{2} q \frac{\gamma q_{x} \mathbf{x}+q_{y} / \gamma \mathbf{y}}{\gamma^{2} q_{x}^{2}+q_{y}^{2} / \gamma^{2}} e^{-i \mathbf{q} \cdot \mathbf{r}} \text {. }
$$

To proceed in our derivation of Eq. (18) we will also need to use the following identity:

$$
\sum_{v} e^{i \mathbf{k} \cdot \mathbf{R}_{v}}=(2 \pi)^{2} n \sum_{\mathbf{K}} \delta_{2}(\mathbf{k}-\mathbf{K}),
$$

where $n=B / \phi_{0}=B \kappa / 2 \pi=\widetilde{B} \widetilde{\kappa} / 2 \pi$ is the numbers of fluxoids.

Upon using Eqs. (17) and (A3) we find 


$$
\begin{aligned}
\eta & =\operatorname{Re}\left[2 \int \frac{d^{2} q}{2 \pi} \frac{i \mathbf{q} \cdot \mathbf{s}_{0}}{\gamma^{2} q_{x}^{2}+q_{y}^{2}+q_{y}^{2} / \gamma^{2}} e^{-i \mathbf{q} \cdot \mathbf{r}} \sum_{v} e^{i(\mathbf{k}-\mathbf{q}-\mathbf{K}) \cdot \mathbf{R}_{v}}\right] \\
& =\operatorname{Re}\left[2 \int \frac{d^{2} q}{2 \pi} \frac{i \mathbf{q} \cdot \mathbf{s}_{0}}{\gamma^{2} q_{x}^{2}+q_{y}^{2} / \gamma^{2}} e^{-i \mathbf{q} \cdot \mathbf{r}(2 \pi)^{2} n} \sum_{\mathbf{K}} \delta_{2}(\mathbf{k}-\mathbf{q}+\mathbf{K})\right] \\
& =\operatorname{Re}\left[2 \widetilde{B} \widetilde{\kappa} \sum_{\mathbf{K}} \frac{i(\mathbf{k}+\mathbf{K}) \cdot \mathbf{s}_{0} e^{i(\mathbf{k}+\mathbf{K}) \cdot \mathbf{r}}}{\left[\gamma^{2}\left(k_{x}+K_{x}\right)^{2}+\left(k_{y}+K_{y}\right)^{2} / \gamma^{2}\right]}\right] .
\end{aligned}
$$

\section{APPENDIX B}

Upon using Eq. (34) and local averages, we have

$$
\begin{aligned}
& \langle\omega\rangle=\langle\omega\rangle \text {, } \\
& \frac{1}{2}\left\langle\omega^{2}\right\rangle=\frac{1}{2} \beta\langle\omega\rangle^{2}\left\langle\left[1+(\bar{\eta}+\varphi)^{2}\right]\right\rangle, \\
& \sum_{i, j} \mu_{i j} \frac{1}{4 \kappa^{2} \omega} \frac{\partial \omega}{\partial x_{i}} \frac{\partial \omega}{\partial x_{j}}=\frac{1}{4 \kappa^{2} \omega}\left[\gamma^{4}\left[\frac{\partial \omega}{\partial x}\right]^{2}+\left(\frac{\partial \omega}{\partial y}\right)^{2}\right] \\
& =\frac{1}{4 \kappa^{2} \omega_{l}(1+\varphi)}\left\{(1+\varphi)^{2}\left[\gamma^{4}\left[\frac{\partial \omega_{l}}{\partial x}\right]^{2}+\left(\frac{\partial \omega_{l}}{\partial y}\right]^{2}\right]+2(1+\varphi) \omega_{l}\left[\gamma^{4} \frac{\partial \omega_{l}}{\partial x} \frac{\partial \varphi}{\partial x}+\frac{\partial \omega_{l}}{\partial y} \frac{\partial \varphi}{\partial y}\right]\right. \\
& \left.+\omega_{l}^{2}\left[\gamma^{4}\left(\frac{\partial \varphi}{\partial x}\right)^{2}+\left(\frac{\partial \varphi}{\partial y}\right)^{2}\right]\right\} \\
& =\frac{\gamma^{2}}{4 \kappa^{2}}\left\{(1+\varphi)\left[2 \widetilde{B} \widetilde{\kappa} \omega_{l}+\gamma^{2} \frac{\partial^{2} \omega_{l}}{\partial x^{2}}+\frac{1}{\gamma^{2}} \frac{\partial^{2} \omega_{l}}{\partial y^{2}}\right]+2\left[\gamma^{2} \frac{\partial \omega_{l}}{\partial x} \frac{\partial \varphi}{\partial x}+\frac{1}{\gamma^{2}} \frac{\partial \omega_{l}}{\partial y} \frac{\partial \varphi}{\partial y}\right)\right. \\
& \left.+\frac{\omega_{l}}{(1+\varphi)}\left[\gamma^{2}\left[\frac{\partial \varphi}{\partial x}\right]^{2}+\frac{1}{\gamma^{2}}\left[\frac{\partial \varphi}{\partial y}\right]^{2}\right]\right\} \\
& \left\langle\sum_{i, j} \mu_{i j} \frac{1}{4 \kappa^{2} \omega} \frac{\partial \omega}{\partial x_{i}} \frac{\partial \omega}{\partial x_{j}}\right\rangle=\frac{\widetilde{B}}{2 \widetilde{\kappa} \gamma^{2}}\langle\omega\rangle+\frac{\gamma^{2}\langle\omega\rangle}{4 \kappa^{2}}\left\langle\left[\varphi\left[\gamma^{2} \frac{\partial^{2} \bar{\eta}}{\partial x^{2}}+\frac{1}{\gamma^{2}} \frac{\partial^{2} \bar{\eta}}{\partial y^{2}}\right]+2\left(\gamma^{2} \frac{\partial \bar{\eta}}{\partial x} \frac{\partial \varphi}{\partial x}+\frac{1}{\gamma^{2}} \frac{\partial \bar{\eta}}{\partial y} \frac{\partial \varphi}{\partial y}\right)\right.\right. \\
& \left.\left.+\gamma^{2}\left(\frac{\partial \varphi}{\partial x}\right)^{2}+\frac{1}{\gamma^{2}}\left(\frac{\partial \varphi}{\partial y}\right)^{2}\right]\right\rangle
\end{aligned}
$$

$$
\begin{aligned}
& \sum_{i, j} \mu_{i j} \omega Q_{B}^{i} Q_{B}^{j}=\omega\left[\gamma^{4}\left(Q_{B}^{x}\right)^{2}+\left(Q_{B}^{y}\right)^{2}\right] \\
& =\frac{\omega}{4 \widetilde{\kappa}^{2} \omega_{l}^{2}}\left[\left(\frac{\partial \omega_{l}}{\partial x}\right)^{2}+\frac{1}{\gamma^{4}}\left(\frac{\partial \omega_{l}}{\partial y}\right)^{2}\right] \\
& =\frac{(1+\varphi)}{4 \widetilde{\kappa}^{2} \gamma^{2}}\left[2 \widetilde{B} \widetilde{\kappa} \omega_{l}+\gamma^{2} \frac{\partial^{2} \omega_{l}}{\partial^{2} x^{2}}+\frac{1}{\gamma^{2}} \frac{\partial^{2} \omega_{l}}{\partial y^{2}}\right] \text {, } \\
& \left\langle\sum_{i, j} \mu_{i j} \omega Q_{B}^{i} Q_{B}^{j}\right\rangle=\frac{\widetilde{B}}{2 \widetilde{\kappa} \gamma^{2}}\langle\omega\rangle+\frac{\langle w\rangle}{4 \widetilde{\kappa}^{2} \gamma^{2}}\left\langle\varphi\left[\gamma^{2} \frac{\partial^{2} \bar{\eta}}{\partial x^{2}}+\frac{1}{\gamma^{2}} \frac{\partial^{2} \bar{\eta}}{\partial y^{2}}\right]\right\rangle, \\
& h_{B z} h_{z}=\frac{1}{4 \widetilde{\kappa}^{2}}(\langle\omega\rangle-\omega+\langle\omega\rangle \varphi)\left(\langle\omega\rangle-\omega+\varphi+\frac{\gamma^{2}\langle\omega\rangle \bar{\eta}}{\widetilde{k}^{2}+\gamma^{2}\langle\omega\rangle}\right) \text {, } \\
& \left\langle h_{B z} h_{z}\right\rangle=\frac{1}{4 \widetilde{\kappa}^{2}}\left\langle\omega^{2}-\langle\omega\rangle^{2}\left[1+(\bar{\eta}+\varphi)^{2}-\frac{\bar{\eta}^{2} \widetilde{k}^{2}}{\tilde{k}^{2}+\gamma^{2}\langle\omega\rangle}\right]\right\rangle \text {. }
\end{aligned}
$$


The transverse component of the magnetic field $\mathbf{h}_{\perp}$ may be found by solving $\nabla^{2} h_{x}=\widetilde{\gamma}^{2} \partial^{2} \omega / \partial y^{2}$ and $\nabla^{2} h_{y}$ $=-\widetilde{\gamma}^{2} \partial^{2} \omega / \partial x \partial y$ whose solution is given by Eqs. (25) and (26) with $\bar{\eta}$ replaced by $(\bar{\eta}+\varphi)$. We then find

$\left\langle h_{\perp}^{2}\right\rangle=\frac{1}{4 \widetilde{\kappa}^{2}} 2 \widetilde{\kappa}^{2} \widetilde{\gamma}^{2} \beta_{1}(\beta-1)\langle\omega\rangle^{2}\left\langle\left[1+(\bar{\eta}+\varphi)^{2}\right]\right\rangle$, where

$$
\frac{\beta_{1}}{2} \sum_{\mathbf{K}(\neq 0)}\left|\omega_{\mathbf{K}}\right|^{2}=\sum_{\mathbf{K}(\neq 0)} \frac{K_{y}^{2}}{K^{2}}\left|\omega_{\mathbf{K}}\right|^{2} .
$$

Now, if we integrate by parts the third term of the right-hand side of Eq. (B3) and then using the periodicity of $\bar{\eta}$ and $\varphi$ we arrive at Eq. (38).
*Present address: Department of Theoretical Physics, University of Manchester, Manchester, M13 9PL, U.K.

${ }^{1}$ M. Prohammer, Physica C 157, 4 (1989).

${ }^{2}$ V. G. Kogan and J. R. Clem, Phys. Rev. B 24, 2497 (1981).

${ }^{3}$ R. A. Klemm and J. R. Clem, Phys. Rev. B 21, 1868 (1981); R. A. Klemm, ibid. 38, 6641 (1988); 41, 117 (1990).

${ }^{4}$ K. G. Petzinger and G. A. Warren, Phys. Rev. B 42, 2023 (1990).

${ }^{5}$ E. H. Brandt, J. Low Temp. Phys. 28, 263 (1977).

${ }^{6}$ A. Houghton, R. A. Pelcovits, and A. Sudb $\varnothing$, Phys. Rev. B 40, 6763 (1989).

${ }^{7}$ V. G. Kogan, J. Low Temp. Phys. 20, 103 (1975).
${ }^{8}$ L. J. Campbell, M. M. Doria, and V. G. Kogan, Phys. Rev. B 38, 2439 (1988).

${ }^{9}$ V. G. Kogan and L. J. Campbell, Phys. Rev. Lett. 62, 1552 (1989).

${ }^{10}$ This problem has already been solved in Ref. 6 for the particular case in which the magnetic field is along $\hat{\mathbf{c}}$-crystal axes.

${ }^{11}$ Equation (37) has already been addressed by M. A. Moore (unpublished).

${ }^{12}$ R. Labusch, Phys. Status Solidi 32, 439 (1969).

${ }^{13}$ G. J. Dolan, F. Holtzberg, C. Feild, and T. R. Dinger, Phys. Rev. Lett. 62, 2184 (1989). 